\title{
Mortar Finite Elements with Dual Lagrange Multipliers: Some Applications ${ }^{\star}$
}

\author{
Bishnu P. Lamichhane and Barbara I. Wohlmuth \\ University of Stuttgart, Institute of Applied Analysis and Numerical Simulation \\ http://www .ians . uni-stuttgart.de/nmh, \{lamichhane, wohlmuth\}@mathematik. \\ uni-stuttgart.de
}

\begin{abstract}
Summary. Domain decomposition techniques provide a powerful tool for the numerical approximation of partial differential equations. We consider mortar techniques with dual Lagrange multiplier spaces to couple different discretization schemes. It is well known that the discretization error for linear mortar finite elements in the energy norm is of order $h$. Here, we apply these techniques to curvilinear boundaries, nonlinear problems and the coupling of different model equations and discretizations.
\end{abstract}

\section{Introduction}

The numerical approximation of partial differential equations is often a challenging task. When different physical models should be used in different subregions, a suitable discretization scheme has to be used in each region. Mortar methods yield efficient and flexible coupling techniques for different discretization schemes. The central idea of mortar methods is to decompose the domain of interest into non-overlapping subdomains and impose a weak continuity condition across the interface by requiring that the jump of the solution is orthogonal to a suitable Lagrange multiplier space, see Bernardi et al. [1993, 1994]. Here, we work with mortar techniques and dual Lagrange multiplier spaces. These non-standard Lagrange multipliers show the same qualitative a priori estimates and quantitative numerical results as the standard ones and yield locally supported basis functions for the constrained space leading to a cheaper numerical realization, see Wohlmuth [2001]. This paper is concerned with applications of mortar methods to couple different physical models in different simulation regions. In the next section, we apply mortar methods to couple compressible and nearly incompressible materials in linear elasticity. In Section 3, the linear Laplace operator is coupled with the non-linear $p$-Laplace operator. Finally in Section 4, we show an application to an elastoacoustic problem, and a generalized eigenvalue problem has to be solved. For

\footnotetext{
^ This work was supported in part by the Deutsche Forschungsgemeinschaft, SFB 404, C12.
} 
all our models, we provide numerical results. The weak coupling in terms of dual Lagrange multipliers results in a diagonal matrix on the slave side. As a consequence, the Lagrange multiplier can be eliminated locally, and optimal multigrid methods can be applied to the resulting positive definite system.

\section{Compressible and Nearly Incompressible Materials}

In this section, we consider a problem in linear elasticity with two different materials in two subdomains, one of them being nearly incompressible. We assume that the domain $\Omega \subset \mathbb{R}^{2}$ is decomposed into two non-overlapping subdomains $\Omega_{1}$ and $\Omega_{2}$ with a common interface $\bar{\Gamma}=\bar{\Omega}_{1} \cap \bar{\Omega}_{2}$, and the subdomain $\Omega_{1}$ is occupied with a nearly incompressible material having a very large Lamé parameter $\lambda_{1}$. It is well-known that standard low order finite elements for nearly incompressible materials suffer from locking, see Babuška and Suri [1992], and various approaches have been introduced to improve the numerical results. Working with a mixed formulation on $\Omega_{1}$, see, e.g., Braess [2001], and standard finite element approach on $\Omega_{2}$, we use mortar techniques with dual Lagrange multipliers to realize the coupling between the two formulations. On each subdomain, we define the space

$$
\mathbf{H}_{*}^{1}\left(\Omega_{k}\right):=\left\{\mathbf{v} \in H^{1}\left(\Omega_{k}\right)^{2}, \mathbf{v}_{\mid \partial \Omega \cap \partial \Omega_{k}}=0\right\}, \quad k=1,2,
$$

and consider the constrained product space

$$
\mathbf{V}:=\left\{\mathbf{v} \in \prod_{k=1}^{2} \mathbf{H}_{*}^{1}\left(\Omega_{k}\right) \mid \int_{\Gamma}[\mathbf{v}] \cdot \psi d \sigma=0, \psi \in \mathbf{M}\right\},
$$

where $\mathbf{M}:=\mathbf{H}^{-\frac{1}{2}}(\Gamma)$ is the Lagrange multiplier space, and $[\mathbf{v}]$ is the jump of $\mathbf{v}$ across $\Gamma$. Introducing an additional unknown $p:=\lambda_{1}$ divu in $\Omega_{1}$, the variational problem is given by: find $[\mathbf{u}, p] \in \mathbf{V} \times L^{2}\left(\Omega_{1}\right)$ such that

$$
\begin{gathered}
a(\mathbf{u}, \mathbf{v})+b(\mathbf{v}, p)=l(\mathbf{v}), \quad \mathbf{v} \in \mathbf{V}, \\
b(\mathbf{u}, q)-\frac{1}{\lambda_{1}} c(p, q)=0, \quad q \in L^{2}\left(\Omega_{1}\right),
\end{gathered}
$$

where $l \in \mathbf{V}^{\prime}$ and

$$
\begin{aligned}
a(\mathbf{u}, \mathbf{v}) & :=\sum_{i=1}^{2} 2 \mu_{i} \int_{\Omega_{i}} \varepsilon(\mathbf{u}): \varepsilon(\mathbf{v}) d x+\lambda_{2} \int_{\Omega_{2}} \operatorname{div} \mathbf{u} \operatorname{div} \mathbf{v} d x, \\
b(\mathbf{v}, q) & :=\int_{\Omega_{1}} \operatorname{div} \mathbf{v} q d x, \quad c(p, q):=\int_{\Omega_{1}} p q d x, \quad \text { and } \quad l(\mathbf{v}):=\int_{\Omega} f \cdot \mathbf{v} d x .
\end{aligned}
$$

Here, $\varepsilon(\mathbf{u})$ is the linear strain tensor. For our example, the domain $\Omega:=$ $\operatorname{conv}\{(0,0),(48,44),(48,60),(0,44)\}$ is decomposed into two subdomains $\Omega_{1}$ 
and $\Omega_{2}$ with $\Omega_{1}:=\operatorname{conv}\{(12,20.25),(36,38.75),(36,50.25),(12,38.75)\}$, and $\Omega_{2}:=\Omega \backslash \bar{\Omega}_{1}$. Here, $\operatorname{conv} \xi$ is the convex hull of the set $\xi$. The decomposition of the domain and the initial triangulation are shown in the left picture of Figure 1. Here, the left boundary of $\Omega$ is fixed and the right boundary is subjected to an in-plane shearing load of $100 \mathrm{~N}$ along the positive $y$-direction. The lower and upper boundaries are set free, and we do not apply any volume force. The material parameters are taken to be $E_{1}=250 \mathrm{~Pa}, E_{2}=80 \mathrm{~Pa}$, $\nu_{1}=0.4999$, and $\nu_{2}=0.35$ to get a nearly incompressible response in $\Omega_{1}$, where $E_{i}$ and $\nu_{i}$ are the Young's modulus and the Poisson ratio on $\Omega_{i}, i=1,2$, respectively. The displacement field is discretized with bilinear finite elements, and the pressure in $\Omega_{1}$ is discretized with piecewise constant functions. The right picture of Figure 1 shows the vertical displacement at $(48,60)$ versus the number of elements. We compare three different numerical schemes. Using standard conforming finite elements (standard) in $\Omega$ does not give satisfying numerical results, whereas the more expensive mixed formulation (mixed) in $\Omega$ provides good results. Our numerical results show that the mortar approach (coupled) is almost as good as the mixed formulation and significantly better than the standard one.
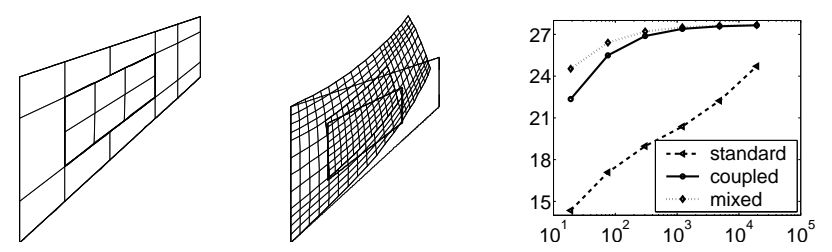

Fig. 1. Decomposition of the domain and initial triangulation (left), distorted grid on level 2 (middle), and vertical tip displacement versus number of elements (right)

\section{The Laplace and the $p$-Laplace Operator}

In this section, we consider the coupling of a linear and a non-linear model. The linear model is described by a Poisson equation, and we use the $p$-Laplacian for the non-linear model. Here, we decompose the domain $\Omega:=(-1,1) \times(-1,1)$ into four non-overlapping subdomains defined by $\Omega_{1}:=(-1,0) \times(-1,0)$, $\Omega_{2}:=(0,1) \times(-1,0), \Omega_{3}:=(-1,0) \times(0,1)$ and $\Omega_{4}:=(0,1) \times(0,1)$. We have given the decomposition of the domain and the initial triangulation in the left picture of Figure 2. We consider the Poisson equation $-\nabla \cdot(\alpha \nabla) u=f$ in $\Omega_{1}$ and $\Omega_{4}$ and the $p$-Laplacian $-\nabla \cdot\left(\alpha|\nabla u|^{p-2} \nabla u\right)=f$ in $\Omega_{2}$ and $\Omega_{3}$. The $p$-Laplace equation occurs in the theory of two-dimensional plasticity under longitudinal shear or in the diffusion problem with non-linear diffusivity, see Atkinson and Champion [1984], and we are considering here different material models in different subdomains. For the regularity of the solutions and error estimates of the p-Laplacian, we refer to Liu and Barret [1993] and Liu and Yan 
[2001]. Let $\mathcal{T}_{h_{k}}$ be a shape regular simplicial triangulation on $\Omega_{k}$ with mesh-
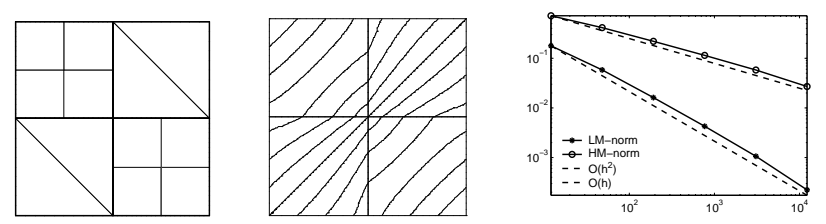

Fig. 2. Decomposition of the domain and initial triangulation (left), isolines of the solution (middle) and discretization errors versus number of elements (right)

sizes bounded by $h_{k}$, and $\mathcal{S}\left(\Omega_{k}, \mathcal{T}_{h_{k}}\right)$ stands for the space of linear conforming finite elements in the subdomain $\Omega_{k}$ associated with the triangulation $\mathcal{T}_{h_{k}}$ satisfying the Dirichlet boundary conditions on $\partial \Omega_{k} \cap \partial \Omega, k=1, \cdots, 4$. Then, the unconstrained finite element space $X_{h}$ is given by $X_{h}:=\prod_{k=1}^{4} \mathcal{S}\left(\Omega_{k}, \mathcal{T}_{h_{k}}\right)$. The interface $\Gamma:=\{(0, y),-1<y<1\} \cup\{(x, 0),-1<x<1\}$ inherits its one-dimensional triangulation $\mathcal{S}_{\Gamma}$ from the mesh on $\Omega_{2}$ and $\Omega_{3}$. We recall that $(0,0)$ is a crosspoint, and $M_{h}$ does not have any degree of freedom at this point. Now, the Lagrange multiplier space $M_{h}$ is defined on $\Gamma$ and is associated with the triangulation $\mathcal{S}_{\Gamma}$. Assuming $q_{1}:=2, q_{2}:=p, q_{3}:=p$, and $q_{4}:=2$, we can write the weak formulation of the problem as: find $\left(u_{h}, \lambda_{h}\right) \in X_{h} \times M_{h}$ such that

$$
\begin{aligned}
a\left(u_{h}, v\right)+b\left(v, \lambda_{h}\right) & =l(v), & & v \in X_{h}, \\
b\left(u_{h}, \mu\right) & =0, & & \mu \in M_{h},
\end{aligned}
$$

where $a(u, v):=\sum_{i=1}^{4} \int_{\Omega_{i}} \alpha|\nabla u|^{q_{i}-2} \nabla u \cdot \nabla v d x, b(v, \mu):=\int_{\Gamma}[v] \mu d \sigma$, and $l(v):=\int_{\Omega} f v d x$. If $\alpha>0$, and the right hand side function $f$ is sufficiently smooth, we can show by monotonicity techniques that the problem (1) has a unique solution, see Liu [1999]. However, the regularity of the solution is not known. Let $u_{h}:=\sum_{k=1}^{n} u_{k} \phi_{k}$ and $\lambda_{h}:=\sum_{k=1}^{n_{s}} \lambda_{k} \mu_{k}$, where $n$ and $n_{s}$ are the dimensions of $X_{h}$ and $M_{h}$, respectively. Suppose $w=\left(u_{1}, \cdots, u_{n}, \lambda_{1}, \cdots, \lambda_{n_{s}}\right)$ be a vector. Now, we define $F(w):=\left(F_{1}(w), F_{2}(w)\right)^{T}$ with

$$
F_{1}(w):=\left(\begin{array}{c}
a\left(u_{h}, \phi_{1}\right)+b\left(\phi_{1}, \lambda_{h}\right)-l\left(\phi_{1}\right) \\
\vdots \\
a\left(u_{h}, \phi_{n}\right)+b\left(\phi_{n}, \lambda_{h}\right)-l\left(\phi_{n}\right)
\end{array}\right), \quad F_{2}(w):=\left(\begin{array}{c}
b\left(u_{h}, \mu_{1}\right) \\
\vdots \\
b\left(u_{h}, \mu_{n_{s}}\right)
\end{array}\right) .
$$

The system $F(w)=0$ is a non-linear system if $p \neq 2$, and we apply the Newton method to solve this system. First, we initialize the solution vector $w_{0}$ satisfying the given Dirichlet boundary conditions. Then, we iterate until convergence with

$$
J_{k} \Delta w_{k}=F\left(w_{k}\right),
$$

where $\Delta w_{k}:=w_{k}-w_{k+1}$, and $J_{k}$ is the Jacobian of $F$ evaluated at $w_{k}$. Working with a dual Lagrange multiplier space has the advantage that the 
Jacobian matrix $J_{k}$ has exactly the same form as the saddle point matrix arising from the mortar finite elements with a dual Lagrange multiplier space for the Laplace operator, see Wohlmuth and Krause [2001]. Hence, we can apply the multigrid approach introduced in Wohlmuth and Krause [2001] to solve the linear system on each level. Suppose that $\tilde{\Omega}_{l}:=\Omega_{1} \cup \Omega_{4}$, and $\tilde{\Omega}_{p}:=\Omega_{2} \cup \Omega_{3}$. In our numerical example, we choose $\alpha=0.5$ in $\tilde{\Omega}_{l}$, and $\alpha=1$ in $\tilde{\Omega}_{p}, p=1.5$, and $f=0$. For boundary conditions, we set $u(-1,-1)=$ $u(1,1)=0, u(-1,1)=1$ and $u(1,-1)=-1$, and the Dirichlet boundary condition on $\partial \Omega$ is imposed by taking the linear combination of them in between. Here, we do not have the exact solution. To get the approximation of the discretization errors, we compute a reference solution $u_{r e f}$ at a fine level and compare it with the solution $u_{h}$ at each level until $h_{r e f} \leq 2 h$. We have given the discretization errors in the $L M$ - and $H M$ - norms defined by

$\|v\|_{L M}:=\|v\|_{L^{2}\left(\tilde{\Omega}_{l}\right)}+\|v\|_{L^{p}\left(\tilde{\Omega}_{p}\right)}$, and $\quad\|v\|_{H M}:=|v|_{W^{1,2}\left(\tilde{\Omega}_{l}\right)}+|v|_{W^{1, p}\left(\tilde{\Omega}_{p}\right)}$

in the right picture of Figure 2, and the isolines of the solution are given in the middle. Although the regularity of the solution is not known, we get convergence of order $h^{2}$ in the $L M$-norm and of order $h$ in the $H M$-norm.

\section{Application to an Elasto-Acoustic Problem}

In this section, we show the application of mortar finite element methods for an elasto-acoustic problem. We consider the situation that the fluid is completely surrounded by the structure. The problem is described by a linear elastic structure occupying a subdomain $\Omega_{S} \subset \mathbb{R}^{2}$ coupled with an irrotational fluid in $\Omega_{F} \subset \mathbb{R}^{2}$. The interface $\Gamma\left(=\partial \Omega_{S} \cap \partial \Omega_{F}\right)$ separates the fluid and solid regions. Given the fluid-density $\rho_{F}$, the solid-density $\rho_{S}$, and the acoustic speed $c$, we seek the frequency $\omega$, the velocity-field $\mathbf{u}$, and the pressure $p$ such that

$$
\begin{aligned}
& \nabla p-\omega^{2} \rho_{F} \mathbf{u}_{F}=\mathbf{0} \text { in } \Omega_{F}, \\
& p+c^{2} \rho_{F} \nabla \cdot \mathbf{u}_{F}=0 \text { in } \Omega_{F}, \\
& \nabla \cdot \sigma\left(\mathbf{u}_{S}\right)+\omega^{2} \rho_{S} \mathbf{u}_{S}=\mathbf{0} \quad \text { in } \Omega_{S} \\
& \mathbf{u}_{S}=\mathbf{0} \quad \text { on } \Gamma_{D}, \\
& \sigma\left(\mathbf{u}_{S}\right) \cdot \mathbf{n}_{S}=\mathbf{0} \text { on } \Gamma_{N}, \\
& \sigma_{n}\left(\mathbf{u}_{S}\right)+p=0, \quad \sigma_{t}\left(\mathbf{u}_{S}\right)=\mathbf{0}, \quad \text { and } \quad\left(\mathbf{u}_{F}-\mathbf{u}_{S}\right) \cdot \mathbf{n}=0 \quad \text { on } \quad \Gamma \text {. }
\end{aligned}
$$

Here, $\sigma$ is the usual stress tensor from linear elasticity, $\sigma_{n}=\mathbf{n} \cdot(\sigma \cdot \mathbf{n})$ is the normal stress on $\Gamma$, and $\sigma_{t}=\sigma \cdot \mathbf{n}-\sigma_{n} \mathbf{n}$ is the tangential traction vector on $\Gamma$, where $\mathbf{n}$ is the outward normal to $\Omega_{F}$ on $\Gamma$. This problem has become a subject of different papers, see, e.g., Hansbo and Hermansson [2003], Bermúdez and Rodríguez [1994], Alonso et al. [2001]. We introduce the following function spaces to formulate our problem in the weak form 
$X:=\mathbf{H}\left(\operatorname{div}, \Omega_{F}\right) \times \mathbf{H}_{\Gamma_{D}}^{1}\left(\Omega_{S}\right)$, and $\mathbf{V}:=\left\{\left(\mathbf{u}_{F}, \mathbf{u}_{S}\right) \in X,[\mathbf{u}] \cdot \mathbf{n}=0\right.$ on $\left.\Gamma\right\}$,

where

$$
\begin{aligned}
\mathbf{H}\left(\operatorname{div}, \Omega_{F}\right) & :=\left\{\mathbf{v} \in L^{2}\left(\Omega_{F}\right)^{2},\|\nabla \cdot \mathbf{v}\|_{L^{2}\left(\Omega_{F}\right)}<\infty\right\}, \\
\mathbf{H}_{\Gamma_{D}}^{1}\left(\Omega_{S}\right) & :=\left\{\mathbf{v} \in H^{1}\left(\Omega_{S}\right)^{2}, \mathbf{v}_{\left.\right|_{\Gamma_{D}}}=0\right\}, \quad \text { and } \quad[\mathbf{u}]:=\left(\mathbf{u}_{F}-\mathbf{u}_{S}\right) .
\end{aligned}
$$

The weak form of the continuous problem is: find $\mathbf{u} \in \mathbf{V}$ and $\omega \in \mathbb{R}$ such that

$$
\begin{aligned}
a(\mathbf{u}, \mathbf{v}) & =\omega^{2} m(\mathbf{u}, \mathbf{v}), \quad \mathbf{v} \in \mathbf{V}, \quad \text { where } \\
a(\mathbf{u}, \mathbf{v}) & :=\left(\rho_{F} c^{2} \nabla \cdot \mathbf{u}_{F}, \nabla \cdot \mathbf{v}_{F}\right) \Omega_{F}+\left(\sigma\left(\mathbf{u}_{S}\right), \epsilon\left(\mathbf{v}_{S}\right)\right)_{\Omega_{S}}, \quad \text { and } \\
m(\mathbf{u}, \mathbf{v}) & :=\left(\omega^{2} \rho_{F} \mathbf{u}_{F}, \mathbf{v}_{F}\right)_{\Omega_{F}}+\left(\omega^{2} \rho_{S} \mathbf{u}_{S}, \mathbf{v}_{S}\right)_{\Omega_{S}} .
\end{aligned}
$$

Here, $\epsilon\left(\mathbf{v}_{S}\right)$ is the linear strain tensor and is related to the stress tensor by Hooke's law, i.e., $\sigma_{i j}\left(\mathbf{v}_{S}\right)=2 \mu \epsilon_{i j}\left(\mathbf{v}_{S}\right)+\lambda \sum_{k=1}^{2} \epsilon_{k k}\left(\mathbf{v}_{S}\right) \delta_{i j}, i, j=1,2$. Let $\mathcal{T}_{h_{s}}$ and $\mathcal{T}_{h_{f}}$ be shape regular simplicial triangulations on $\Omega_{S}$ and $\Omega_{F}$, respectively, and $\Gamma$ inherits its triangulation $\mathcal{S}_{\Gamma}$ from the side of $\Omega_{F}$. It is a well-known fact that if standard Lagrangian finite elements are used to discretize the fluid, it will give rise to spurious eigensolutions with positive eigenvalues interspersed among the 'real' ones, and a possible remedy of this problem is to use RaviartThomas elements in the fluid domain, see Bermúdez et al. [1995]. Therefore, we discretize the fluid domain with Raviart-Thomas elements of lowest order:

$$
R T_{0}:=\left\{\mathbf{u} \in \mathbf{H}\left(\operatorname{div}, \Omega_{F}\right): \mathbf{u}_{\left.\right|_{K}}=(a+b x, c+b y), K \in \mathcal{T}_{h_{f}}, \quad a, b, c \in \mathbb{R}\right\},
$$

and the solid domain with Lagrangian finite elements of lowest order:

$$
W_{h}^{D}:=S_{D}\left(\Omega_{S}, \mathcal{T}_{h_{s}}\right) \times S_{D}\left(\Omega_{S}, \mathcal{T}_{h_{s}}\right)
$$

where $S_{D}\left(\Omega_{S}, \mathcal{T}_{h_{s}}\right)$ is the finite element space on $\Omega_{S}$ satisfying the Dirichlet boundary condition on $\Gamma_{D}$. The kinematic constraint can be imposed by piecewise constant Lagrange multipliers yielding a uniform inf-sup condition. Suppose $X_{h}:=R T_{0} \times W_{h}^{D}$, and $M_{h}:=\left\{\mu_{h} \in L^{2}(\Gamma):\left.\mu_{h}\right|_{e} \in \mathcal{P}_{0}(e), e \in \mathcal{S}_{\Gamma}\right\}$. Now the finite element space can be written as

$$
\mathbf{V}_{h}:=\left\{\left(\mathbf{u}_{h F}, \mathbf{u}_{h S}\right) \in X_{h}, \int_{\Gamma}\left[\mathbf{u}_{h}\right] \cdot \mathbf{n} \mu_{h} d \sigma=0, \mu_{h} \in M_{h}\right\} .
$$

The discrete problem reads: find $\mathbf{u}_{h} \in \mathbf{V}_{h}$, and $\omega_{h} \in \mathbb{R}$ such that

$$
a\left(\mathbf{u}_{h}, \mathbf{v}_{h}\right)=\omega_{h}^{2} m\left(\mathbf{u}_{h}, \mathbf{v}_{h}\right), \quad \mathbf{v}_{h} \in \mathbf{V}_{h} .
$$

Remark 1. We remark that the Lagrange multiplier $\lambda_{h}$ approximates the pressure on the interface $\Gamma$. The Lagrange multipliers are associated with the onedimensional mesh inherited from the triangulation on the fluid domain. Due to the special structure of the support of the nodal basis functions of $R T_{0}$ and $M_{h}$, the degree of freedom corresponding to the Lagrange multiplier can locally be eliminated by inverting a diagonal mass matrix. 
In Alonso et al. [2001], an adaptive finite element scheme is analyzed to solve the fluid-structure vibration problem, where the kinematic constraint is imposed by means of piecewise constant Lagrange multiplier. Following this technique, we arrive at the same mortar setting as we discuss here. Now, we consider the domain $\Omega:=\left\{(x, y) \in \mathbb{R}^{2}, x^{2}+y^{2}<1\right\}$ decomposed into two subdomains $\Omega_{S}$ and $\Omega_{F}$ with $\Omega_{F}:=\left\{(x, y) \in \mathbb{R}^{2}, x^{2}+y^{2}<0.6\right\}$, and $\Omega_{S}:=\Omega \backslash \bar{\Omega}_{F}$. Here, $\Gamma_{D}=\left\{(\cos \theta, \sin \theta), \frac{5 \pi}{4} \leq \theta \leq \frac{7 \pi}{4}\right\}$. We have used the following parameters in our numerical example: $\rho_{F}=1000 \mathrm{~kg} / \mathrm{m}^{3}, c=1430 \mathrm{~m} / \mathrm{s}$, $\rho_{S}=7700 \mathrm{~kg} / \mathrm{m}^{3}, E=144 \mathrm{GPa}$, and $\nu=0.35$. The first three consecutive eigenmodes along with the pressure in the fluid domain and the distorted grids in the solid domain are shown in Figure 3. We note that $\Gamma$ defines a curvilinear interface. To evaluate the weak coupling, we commit an additional variational crime by projecting the mesh of the structure side to the mesh on the fluid side.
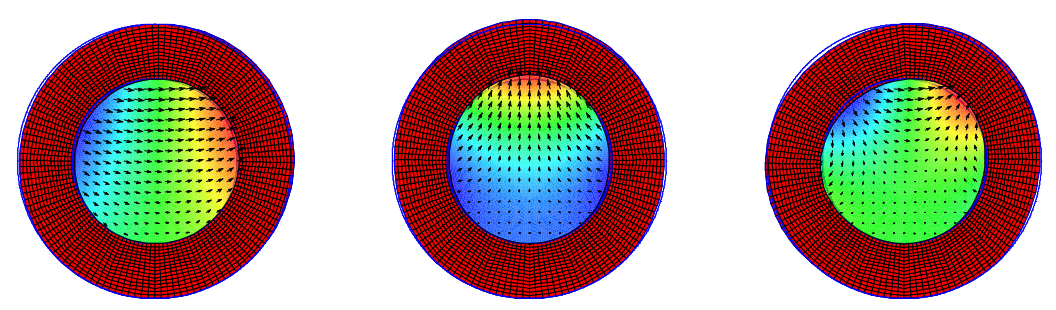

Fig. 3. The first, second and the third eigenmodes corresponding to the eigenvalues $809.1481,1980.7519$ and $3606.3907(\mathrm{rad} / \mathrm{s})$

The second numerical example is taken from Bermúdez and Rodríguez [1994]. The domain $\Omega:=(0,1.5) \times(0,1.5)$ is decomposed into two subdomains $\Omega_{S}$ and $\Omega_{F}$ with $\Omega_{F}:=(0.25,1.25) \times(0.25,1.25), \Omega_{S}:=\Omega \backslash \bar{\Omega}_{F}$, and $\Gamma_{D}=\left\{(x, 0) \in \mathbb{R}^{2}, 0 \leq x \leq 1.5\right\}$. We have used the same physical parameters as in the previous example. The computed eigenfrequencies (in rad/s) along with the extrapolated ones referred to as 'Exact' in Bermúdez and Rodríguez [1994] are given in Table 1.

Table 1. The computed eigenfrequencies using mortar techniques compared with the extrapolated eigenfrequencies ('Exact') in Bermúdez and Rodríguez [1994]

\begin{tabular}{ccc}
\hline Eigenmodes Computed Eigenfrequencies & 'Exact' \\
\hline 1 & 648.1847 & 641.837 \\
2 & 2147.3593 & 2116.398 \\
3 & 3419.5020 & 3201.475 \\
4 & 3885.9022 & 3804.124 \\
5 & 4214.0865 & 4211.620 \\
6 & 4699.6782 & 4687.927 \\
\hline
\end{tabular}




\section{References}

A. Alonso, A. D. Russo, C. Otero-Souto, C. Padra, and R. Rodríguez. An adaptive finite element scheme to solve fluid-structure vibration problems on non-matching grids. Computing and Visualization in Science, 4:67-78, 2001.

C. Atkinson and C. R. Champion. Some boundary-value problems for the equation $\nabla \cdot\left(|\nabla \phi|^{N}\right)=0$. Quart. J. Mech. Appl. Math., 37:401-419, 1984.

I. Babuška and M. Suri. On locking and robustness in the finite element method. SIAM J. Numer. Anal., 29:1261-1293, 1992.

A. Bermúdez, R. Duran, M. Muschietti, R. Rodríguez, and J. Solomin. Finite element vibration analysis of fluid-solid systems without spurious modes. SIAM J. Numer. Anal., 32:1280-1295, 1995.

A. Bermúdez and R. Rodríguez. Finite element computation of the vibration modes of a fluid-solid system. Comput. Meth. in Appl. Mech. and Engrg., 119:355-370, 1994.

C. Bernardi, Y. Maday, and A. Patera. Domain decomposition by the mortar element method. In H. K. et al., editor, Asymptotic and numerical methods for partial differential equations with critical parameters, pages 269-286. Reidel, Dordrecht, 1993.

C. Bernardi, Y. Maday, and A. Patera. A new nonconforming approach to domain decomposition: the mortar element method. In H. B. et al., editor, Nonlinear partial differential equations and their applications, pages 13-51. Paris, 1994.

D. Braess. Finite Elements. Theory, fast solver, and applications in solid mechanics. Cambridge Univ. Press, Second Edition, 2001.

P. Hansbo and J. Hermansson. Nitsche's method for coupling non-matching meshes in fluid-structure vibration problems. Comput. Mech., 32:134-139, 2003.

W. Liu. Degenerate quasilinear elliptic equations arising from bimaterial problems in elastic-plastic mechanics. Nonlinear Analysis, 35:517-529, 1999.

W. Liu and J. Barret. A remark on the regularity of the solutions of the p-Laplacian and its application. J. Math. Anal. Appl., 178:470-488, 1993.

W. Liu and N. Yan. Quasi-norm local error estimators for p-Laplacian. SIAM J. Numer. Anal., 39:100-127, 2001.

B. Wohlmuth. Discretization Methods and Iterative Solvers Based on Domain Decomposition, volume 17 of LNCS. Springer Heidelberg, 2001.

B. Wohlmuth and R. Krause. Multigrid methods based on the unconstrained product space arising from mortar finite element discretizations. SIAM J. Numer. Anal., 39:192-213, 2001. 